\title{
Operations of a Microgrid with Renewable Energy Integration and Line Switching
}

\author{
José Luis Ruiz Duarte · Neng Fan
}

March 16, 2018

\begin{abstract}
With the development of new technologies and their integration to the conventional power grid, the smart grid with the capacity of satisfying power demand by large amount of renewable energy is emerging. Microgrid, a small-scale power system with clearly defined electrical boundaries and ability of self-supply, especially by distributed renewable energy, plays a big role in this process. In this paper, we study the operations of a microgrid with solar photovoltaic generators, energy storage system, and power exchanges with main power grid. More specifically, a mixed integer programming model is formulated for decision-making, such as scheduling of generators within the microgrid, islanding operations through line switching and power trades between microgrid and the main grid, charging and discharging operations of storage system, and also line switching within the microgrid, by robust optimization for capturing the uncertainties of solar power generation. To solve the robust optimization formulation, we formulate our model in order to apply the column-andconstraint generation algorithm, and perform numerical experiments on several test cases to validate the proposed model and algorithm.
\end{abstract}

Keywords Microgrid · Renewable energy sources · Strorage systems · Line switching · Robust optimization · Column-and-constraint generation algorithm

\section{Introduction}

Exponential growth in electricity demand has given distributed generation (DG) technology a boost. DG can be defined as electric power generation within distribution networks or on the customer side of the network, and Distributed Resources (DR) as demand- and supply-side resources that can be deployed throughout an electric

J.L. Ruiz Duarte · N. Fan

Department of Systems and Industrial Engineering, University of Arizona, Tucson, AZ 85721

N. Fan

E-mail: nfan@email.arizona.edu 
distribution system to meet the energy and reliability needs of the customers served by that system [1]. DG changes the nature of the distribution system from passive to active [2].

Electricity generation from Renewable Energy Sources (RESs) has gained attention due to an international effort for reaching ambitious targets of $\mathrm{CO}_{2}$ reduction [3], and the advancement in power electronics technology [4] and sofisticated integration schemes that include optimization approach [5]. The development of solar and wind energy resources technology has resulted on more DGs added to the centralized generation system [6]. Compared to conventional DG sources, RESs have the challenge of higher variability and lower efficiency [7]. Nevertheless, an improperly managed high RES penetration may cause network instability [8]. Some authors consider distribution utilities cannot rely solely on RESs, so Energy Storage Systems (ESSs) can be used to facilitate integration of RESs [9]. Researches such as the one conducted by Genoese and Genoese [10] evaluate the economical impact of including ESSs in power systems with integrated RESs. The smart grid initiative calls for integrating significant levels of renewable energy [7].

With growing applications and potential to compete with conventional grids, microgrids are becoming important for energy infrastructure planning [11]. Microgrid technology is an attractive way for electricity consumers to pursue energy efficiency and clean energy in tandem. A microgrid can be defined as a small-scale power system with at least one DR and one load with clearly defined electrical boundaries, the ability of self-supply, and islanding [12], that could prevent the system from a total collapse [13]. Intentional islanding occurs when a grid is divided in two or more parts, and each operates electrically isolated [14]. We consider islanding operations as the ability of the microgrid to operate disconnected from the main grid.

Opportunities introduced by microgrids include improved reliability, improved resiliency, higher power quality reduction in carbon emission, utilization of RES, improved efficiency, cost reduction, and loads supply on remote areas [12]. Management of a microgrid can be more sophisticated and complex than a larger, and has the potential to mitigate the effects of resource uncertainty [15]. By utilizing Information and Communication Technologies (ICT), microgrids can coordinate Distributed Energy Resources (DER) and controllable loads efficiently [16]. A microgrid that is connected to the main grid, can be switched over to the islanded mode and operate autonomously for safety purposes [16], and can manage the import and export of power to the main grid [17]. Controllable DGs in a microgrid are smaller, reflecting an easier switching operation and hence a more flexible scheduling [18]. The optimization of small-scale power systems has important differences from large scale ones [16]: a larger impact and penetration of RESs, the power demand contains some flexibility, and that the microgrid is able to switch between grid-connected mode and islanded mode.

There are different approaches for operations and control of microgrids in the literature. Maknouninejad et al. [8] proposed a multilevel smartgrid control approach using interconnected microgrids, obtaining optimal operations for the DER to increase the overall smartgrid efficiency. Rezvani et al. [19] developed an algorithm that combines Artificial Neural Networks and Genetic Algorithms to find the maximum power point of RES integrated into microgrid. Heymann et al. [20] compared interior- 
point algorithm, a dynamic programming, and mixed integer linear programming (MILP) for islanding mode microgrid management. Wang and Huang [21] developed a two-stage stochastic program for investment and operation under uncertainty of a microgrid. Hytowitz and Hedman [15] developed a co-optimization framework for a microgrid, integrating solar photovoltaic (PV) generation, emergency generation, connection between main and microgrid, and transmission switching on a network topology. Parisio et al. [22] managed uncertainty by embedding MILP into a Model Predictive Control a Model Predictive Control to compensate uncertainty. Kazemzadeh et al. [25] developed a unit commitment model using stochastic programming integrated with risk measures, and robust optimization, in order to compare both methods to deal with random data. Guo and Zhao [16] developed a robust optimization algorithm for energy management including RES, Combined Heat and Power, ESS, and flexible demand. The problem was solved by the Column-andConstraint-Generation (C\&CG) algorithm, following the idea in [23, 24].

In this paper, we develop a model for the optimal operations of a microgrid, including the uncertainty related to RES, specifically solar energy. The more conservative robust optimization is used to deal with solar power generation uncertainty, since we consider that shortages resulting from bad decisions are costly. Another advantage of robust optimization is that, once an optimal solution is found, little or no adjustment needs to be made to the decision variables that are dependent on the uncertainty [26]. The decisions to be made in our model include scheduling of generators within the microgrid, islanding operations through line switching and power trades between microgrid and the main grid, charging and discharging operations of storage system, and also line switching within the microgrid. To solve the large-scale robust and MILP model, a C\&CG algorithm is utilized to deal with this, and to optimize the operations of the microgrid under the worst-case scenario of renewable output. Compared to existing literature, this paper considers more complicated smart grid technologies and applies robust optimization for microgrid operations.

The main contribution of this paper consists on a model to optimize microgrid operations of distributed generators, solar PV generators, load demands, ESSs, and its connections to the main grid with transmission lines considering a network topology, and its reformulation in order to apply the C\&CG algorithm. The remainder of the paper is organized as follows. In Section 2 the model for optimal scheduling is presented. In Section 3 the problem is reformulated and the solution algorithm is developed. In Section 4 an illustrative example and results are presented. Finally, Section 5 concludes the paper.

\section{Robust optimization model for operations of microgrid and islanding}

\subsection{Nomenclature}

In this paper, we study a microgrid that is connected to the main grid. Within the microgrid, we have distributed generators, solar PV generators, load demands, EES, and its connections to the main grid with transmission lines. The decisions to be made include scheduling of generators within the microgrid, islanding operations through 
Table 1 Sets and Indices

$\mathscr{T}$ Set of time periods for microgrid operations, indexed by $t$

$\mathscr{I} \quad$ Set of buses within microgrid, indexed by $i$

$\mathscr{G}$ Set of generators within microgrid, indexed by $g$ (connected to bus $i_{g} \in I$ )

$\mathscr{E} \quad$ Set of transmission lines, indexed by $e$ (denoted by $e=\left(i_{e}, j_{e}\right)$ with tail bus $i_{e}$ and head bus $j_{e}$ )

$\mathscr{E}^{\prime} \subseteq \mathscr{E} \quad$ Subset of transmission lines between main and microgrid

$\mathscr{S} \quad$ Set of ESS, indexed by $s$ (connected to bus $i_{s} \in I$ )

line switching and power trades between microgrid and the main grid, charging and discharging operations of storage system, power flows and line switching within the microgrid. To concentrate on the microgrid operations we do not consider the operations of the main grid, except for the line switching that causes grid-connected or islanding mode and power trades between microgrid and main grid. Additionally, we assume the main grid is always ready to satisfy the demand of the microgrid in any time and any amount, and also it will accept the excessive energy generation of the microgrid in any time.

The notations, given parameters and problem settings are shown in the following Tables 1, 2, and 3 . 
Table 2 Parameters

\begin{tabular}{|c|c|}
\hline$P_{g}^{\min }, P_{g}^{\max }$ & Lower/upper limits on power output for generation unit $g \in \mathscr{G}$ \\
\hline$T_{g}^{d 0}, T_{g}^{u 0}$ & Minimum time periods generation unit $g \in \mathscr{G}$ must be initially offline/online \\
\hline$\stackrel{g}{T_{g}^{d}}, \stackrel{g}{g}_{g}^{u}$ & $\begin{array}{l}\text { Minimum time periods generation unit } g \in \mathscr{G} \text { must remain offline/online once the } \\
\text { unit is shut down/started up }\end{array}$ \\
\hline$R_{g}^{d}, R_{g}^{u}$ & $\begin{array}{l}\text { Maximum ramp-down and ramp-up rate for generation unit } g \in \mathscr{G} \text { between } \\
\text { adjacent time periods }\end{array}$ \\
\hline$\tilde{R}_{g}^{d}, \tilde{R}_{g}^{u}$ & $\begin{array}{l}\text { Maximum shutdown/startup ramp rates for generation unit } g \in \mathscr{G} \text { for a time period } \\
\text { in which } \mathrm{g} \text { is turned off/on }\end{array}$ \\
\hline$C_{g}^{u}, C_{g}^{d}$ & Fixed startup/shutdown cost for generation unit $g \in \mathscr{G}$ \\
\hline$C_{g}^{p^{\circ}}$ & Production cost function for generation unit $g \in \mathscr{G}$ \\
\hline$F_{e}$ & Capacity of transmission line $e \in \mathscr{E}$ \\
\hline$B_{e}$ & Susceptance of line $e \notin \mathscr{E}^{\prime}$ \\
\hline$S_{s}^{\min }, S_{s}^{\max }$ & Minimum and maximum storage capacity for ESS unit $s \in \mathscr{S}$ \\
\hline$S_{S, 0}$ & Initial State of Charge of the ESS unit $s \in \mathscr{S}$ \\
\hline$\eta_{s}^{+/-}$ & Charging (+) or discharging (-) efficiency of ESS unit $s \in \mathscr{S}$ \\
\hline$R_{s}^{+}, R_{s}^{-}$ & Maximum charging (+) or discharging (-) rate of ESS unit $s \in \mathscr{S}$ \\
\hline$C_{s}^{+}, C_{s}^{-}$ & $\begin{array}{l}\text { Cost related to affected lifetime on ESS unit } s \in \mathscr{S} \text { when energy is being } \\
\text { charging }(+) \text { or discharging }(-)\end{array}$ \\
\hline$\overline{P t}_{t}^{+F}, \overline{P t}_{t}^{-F}$ & $\begin{array}{l}\text { Maximum power sold to }(+) \text {, or purchased from (-) main grid, under firm (F) } \\
\text { contract, at time } t \in \mathscr{T}\end{array}$ \\
\hline$C_{m, t}^{+F}, C_{m, t}^{+N}, C_{m, t}^{-F}, C_{m, t}^{-N}$ & $\begin{array}{l}\text { Cost of power sold to (+), or purchased from (-) main grid, under firm (F) or } \\
\text { non-firm (N) contract, at time } t \in \mathscr{T}\end{array}$ \\
\hline$D_{i, t}$ & Energy demand for load at bus $i \in \mathscr{I}$, at time $t \in \mathscr{T}$ \\
\hline$C_{i, t}^{s h}$ & Cost of shedding one unit of power on bus $i \in \mathscr{I}$ at time $t \in \mathscr{T}$ \\
\hline$P V_{p v, t}^{\text {mean }}$ & Mean value of solar power generation at generator $p v$, at time $t \in \mathscr{T}$ \\
\hline$\sigma_{p v, t}$ & Standard deviation of solar power generation at generator $p v$, at time $t \in \mathscr{T}$ \\
\hline$\overline{P V_{p v}}$ & Maximum capacity of solar power generation at generator $p v$ \\
\hline
\end{tabular}

Table 3 Decision Variables

\begin{tabular}{cl}
\hline$x_{g, t} \in\{0,1\}$ & Binary variable indicating if a generation unit $g \in \mathscr{G}$ is commited $\left(x_{g, t}=1\right)$ \\
& or not $\left(x_{g, t}=0\right)$ at time $t \in \mathscr{T}$ \\
$c_{g, t}^{u}, c_{g, t}^{d}$ & Incurred startup/shutdown cost for a generation unit $g \in \mathscr{G}$ at time $t \in \mathscr{T}$ \\
$p_{g, t}$ & Power generation of generator $g \in \mathscr{G}$ at time $t \in \mathscr{T}$ \\
$f_{e, t}$ & Power flow on line $e \in \mathscr{E}$, at time $t \in \mathscr{T}$ \\
$z_{e, t} \in\{0,1\}$ & Line $e \in \mathscr{E}$ is switched on $\left(z_{e, t}=1\right)$ or off $\left(z_{e, t}=0\right)$, at time $t \in \mathscr{T}$ \\
$\theta_{i_{e}, t}$ & Voltage angle of bus $i$ on line $e \in \mathscr{E}$, at time $t \in \mathscr{T}$ \\
$p_{m, t}^{+F}, p_{m, t}^{+N}, p_{m, t}^{-F}, p_{m, t}^{-N}$ & Power sold to $(+)$, or purchased from $(-)$ main grid, under firm (F) or non-firm \\
& (N) contract, at time $t \in \mathscr{T}$ \\
$m_{t} \in\{0,1\}$ & Binary variable indicating if microgrid is connected to main grid (m $\left.m_{t}=1\right)$, \\
& or not $\left(m_{t}=0\right)$, at time $t \in \mathscr{T}$ \\
$y_{s, t}$ & State of Charge on ESS unit $s \in \mathscr{S}$, at time $t \in \mathscr{T}$ \\
$r_{s, t}^{+}, r_{s, t}^{-}$ & Amount of power to charge $(+)$or discharge $(-)$of ESS unit $s \in \mathscr{S}$, at time $t \in \mathscr{T}$ \\
$q_{i, t}$ & Amount of load shed at bus $i \in \mathscr{I}$, at time $t \in \mathscr{T}$ \\
$P V_{p v, t}$ & Photovoltaic power generated at generator $p v$ at time $t \in \mathscr{T}$ \\
& \\
\hline
\end{tabular}


2.2 An optimization model with renewable energy integration

Scheduling of generators within microgrid. As mentioned above, we do not consider the operations of main grid, except for the line switching that cause grid-connected or islanding mode and the power exchange between microgrid and main grid. The set of constraints related to the generation capacity of each generator within the microgrid, and to the decision of turning on or off the generator, are modeled in (1a)-(1m).

$$
\begin{aligned}
& \sum_{t=1}^{T_{g}^{u 0}}\left(1-x_{g, t}\right)=0, \quad \forall g \in \mathscr{G} \\
& \sum_{t=1}^{T_{g}^{d 0}} x_{g, t}=0, \quad \forall g \in \mathscr{G} \\
& \sum_{t^{\prime}=t}^{t+T_{g}^{u}-1} x_{g, t^{\prime}} \geq T_{g}^{u}\left(x_{g, t}-x_{g, t-1}\right), \quad \forall g \in \mathscr{G}, t \in\left\{T_{g}^{u 0}+1, \ldots, T-T_{g}^{u}+1\right\} \\
& \sum_{t^{\prime}=t}^{t+T_{g}^{d}-1}\left(1-x_{g, t^{\prime}}\right) \geq T_{g}^{d}\left(x_{g, t-1}-x_{g, t}\right), \quad \forall g \in \mathscr{G}, t \in\left\{T_{g}^{d 0}+1, \ldots, T-T_{g}^{d}+1\right\} \\
& \sum_{t^{\prime}=t}^{T}\left(x_{g, t^{\prime}}-\left(x_{g, t}-x_{g, t-1}\right)\right) \geq 0, \forall g \in \mathscr{G}, t \in\left\{T-T_{g}^{u}+2, \ldots, T\right\} \\
& \sum_{t^{\prime}=t}^{T}\left(\left(1-x_{g, t^{\prime}}\right)-\left(x_{g, t-1}-x_{g, t}\right)\right) \geq 0, \forall g \in \mathscr{G}, t \in\left\{T-T_{g}^{d}+2, \ldots, T\right\} \\
& c_{g, t}^{u} \geq C_{g}^{u}\left(x_{g, t}-x_{g, t-1}\right), \quad \forall g \in \mathscr{G}, t \in\{2, \ldots, T\} \\
& c_{g, t}^{d} \geq C_{g}^{d}\left(x_{g, t-1}-x_{g, t}\right), \quad \forall g \in \mathscr{G}, t \in\{2, \ldots, T\} \\
& P_{g}^{\text {min }} x_{g, t} \leq p_{g, t} \leq P_{g}^{\text {max }} x_{g, t}, \quad \forall g \in \mathscr{G}, t \in \mathscr{T} \\
& p_{g, t}-p_{g, t-1} \leq R_{g}^{u} x_{g, t-1}+\tilde{R}_{g}^{u}\left(x_{g, t}-x_{g, t-1}\right)+P_{g}^{\max }\left(1-x_{g, t}\right), \\
& \forall g \in \mathscr{G}, t \in\{2, \ldots, T\} \\
& p_{g, t-1}-p_{g, t} \leq R_{g}^{d} x_{g, t}+\tilde{R}_{g}^{d}\left(x_{g, t-1}-x_{g, t}\right)+P_{g}^{\max }\left(1-x_{g, t-1}\right), \\
& \forall g \in \mathscr{G}, t \in\{2, \ldots, T\}
\end{aligned}
$$

Constraints (1a) and (1b) represent the initial online/offline requirements for generation units. Minimum number of time periods the generator must remain online/offline after the minimum online/offline required time is represented on (1c) and (1d). Minimum number of time periods the generator must remain online/offline at the end of the time horizon are represented on (1e) and (1f). Start up and shut down cost computation are modeled in $(1 \mathrm{~g})$ and $(1 \mathrm{~h})$, respectively. A generator can only supply power if it is online, and can only supply an amount within lower and upper limits given by its technical capacity, as modeled in (1i). A generator can change its 
power supply depending on its ramping rate, that determines how fast the generation can be changed hourly, or when the generator is turned on or off; this is modeled in (1j)-(1k). Non-negativity constraints for the power generation and start up and shut down cost variables are modeled in (11). Binary constraints for the decision of the online/offline status of a generator are modeled in $(1 \mathrm{~m})$. For the case of initial time $t=1$, we assume that in period $t=0$, a generator was supplying an amount of $\bar{p}_{g, 0}$, and its online/offline status was $\bar{x}_{g, 0}$; as these are fixed values, they can be considered as parameters. For that case, we add constraints (2a)-(2d).

$$
\begin{aligned}
& p_{g, 1}-\bar{p}_{g, 0} \leq R_{g}^{u} \bar{x}_{g, 0}+\tilde{R}_{g}^{u}\left(x_{g, 1}-\bar{x}_{g, 0}\right)+P_{g}^{\max }\left(1-x_{g, 1}\right), \quad \forall g \in \mathscr{G} \\
& \bar{p}_{g, 0}-p_{g, 1} \leq R_{g}^{d} x_{g, 1}+\tilde{R}_{g}^{d}\left(\bar{x}_{g, 0}-x_{g, 1}\right)+P_{g}^{\max }\left(1-\bar{x}_{g, 0}\right), \quad \forall g \in \mathscr{G} \\
& c_{g, 1}^{u} \geq C_{g}^{u}\left(x_{g, 1}-\bar{x}_{g, 0}\right), \quad \forall g \in \mathscr{G} \\
& c_{g, 1}^{d} \geq C_{g}^{d}\left(\bar{x}_{g, 0}-x_{g, 1}\right), \quad \forall g \in \mathscr{G}
\end{aligned}
$$

Capacity of transmission lines and line switching. In this model, a network approach is utilized. For this case, transmission lines can be seen as the edges of the network. The power flow that is generated on a node $i$ can satisfy the demand on a node $j$ if there is a path that connects nodes $i$ and $j$ through transmission lines. The initial and end nodes of each lines are considered. Initial node is denoted as $i_{e}$ and end node is denoted as $j_{e}$. The dynamics of transmission lines are modeled in the set of constraints $(3 a)-(3 c)$.

$$
\begin{aligned}
& -F_{e} z_{e, t} \leq f_{e, t} \leq F_{e} z_{e, t}, \quad \forall e \in \mathscr{E}, t \in \mathscr{T} \\
& f_{e, t}=B_{e}\left(\theta_{i_{e}, t}-\theta_{j_{e}, t}\right) z_{e, t}, \quad \forall e \notin \mathscr{E}^{\prime}, t \in \mathscr{T} \\
& z_{e, t} \in\{0,1\}, \forall e \in \mathscr{E}, t \in \mathscr{T}
\end{aligned}
$$

The transmission limits given by the physical capacity of transmission lines are modeled in (3a). The Kirchhoff's law, that considers the power flow of a transmission line within the microgrid is proportional to the difference in voltage phase angles at the terminal buses of the line, taking into account the decision of connecting/disconnecting the transmission line is modeled in (3b). The connected/disconnected status of a line is denoted as the variable $z_{e, t}$, and the binary constraint for this variable is modeled in (3c).

Note that $(3 \mathrm{~b})$ involves a nonlinear term. Then, it can be properly reformulated to linearize the terms involved. This is presented in (4). If $z_{e, t}=1,(4)$ reduces to (3b) but removing variable $z_{e, t}$. Otherwise, (4) makes $\theta_{i_{e}, t}-\theta_{j_{e}, t}$ be unrestricted (or bounded by big $M$ constants).

$$
\begin{gathered}
B_{e}\left(\theta_{i_{e}, t}-\theta_{j_{e}, t}\right)-\left(1-z_{e, t}\right) M_{e} \leq f_{e, t} \leq B_{e}\left(\theta_{i_{e}, t}-\theta_{j_{e}, t}\right)+\left(1-z_{e, t}\right) M_{e} \\
\forall e \notin \mathscr{E}^{\prime}, t \in \mathscr{T}
\end{gathered}
$$

Main grid and microgrid trade, and islanding mode operations of microgrid. For the network structure, we assume that main grid is always ready to satisfy the 
demand of the microgrid in any time and any amount, and also it will accept the excessive energy generation of the microgrid in any time. Power can be purchased or sold to the main grid at different rates. The power traded under contract is called firm $(\mathrm{F})$, and the power traded above the limits of the contract are called non-firm $(\mathrm{N})$. The trade prices for both $\mathrm{F}$ and $\mathrm{N}$, sales or purchases are different. The set of constraints for the grid-connection or islanding mode and trades between main and microgrid are modeled in $(5 \mathrm{a})-(5 \mathrm{~g})$.

$$
\begin{aligned}
& \left(p_{m, t}^{-N}+p_{m, t}^{-F}\right)-\left(p_{m, t}^{+N}+p_{m, t}^{+F}\right)=\sum_{e \in \mathscr{E}^{\prime}} f_{e, t}, \quad \forall t \in \mathscr{T} \\
& -\sum_{e \in \mathscr{E}^{\prime}} F_{e}\left(m_{t}\right) \leq p_{m, t}^{-N}+p_{m, t}^{-F} \leq \sum_{e \in \mathscr{E}^{\prime}} F_{e}\left(m_{t}\right), \quad \forall t \in \mathscr{T} \\
& -\sum_{e \in \mathscr{E}^{\prime}} F_{e}\left(m_{t}\right) \leq p_{m, t}^{+N}+p_{m, t}^{+F} \leq \sum_{e \in \mathscr{E}^{\prime}} F_{e}\left(m_{t}\right), \quad \forall t \in \mathscr{T} \\
& p_{m, t}^{+F} \leq \overline{P t}_{t}^{+F}, \forall t \in \mathscr{T} \\
& p_{m, t}^{-F} \leq \overline{P t}_{t}^{-F}, \forall t \in \mathscr{T} \\
& p_{m, t}^{-N}, p_{m, t}^{-F}, p_{m, t}^{+N}, p_{m, t}^{+F} \geq 0, \forall t \in \mathscr{T} \\
& m_{t} \in\{0,1\}, \forall t \in \mathscr{T}
\end{aligned}
$$

The total power flow on the transmission lines between main and microgrid is modeled in (5a). The transmission lines that connect main and microgrid can also be connected/disconnected, that is, microgrid can be in grid-connected or islanding mode, and this affects the power flow on these lines, as formulated in (5b)-(5c). The amount of power that can be traded under firm contract is also modeled (5d)-(5e). The non-negativity constraints for the power traded between main and microgrid are modeled in (5f). The decision of connecting/disconnecting the microgrid from the main grid is denoted as variable $m_{t}$, and its binary constraints are modeled in $(5 \mathrm{~g})$.

Operations of storage systems. ESSs are also considered for this model. The ESSs are helpful to catch the surplus of power generated that are not needed by the loads on a period of time, or also to supply power when it is convenient [27]. This also helps to reduce the effects of the uncertainty associated with random power generation, e.g., solar PV power generation. In this model several ESSs placed in different buses of microgrid are considered. The dynamics of the ESSs are modeled in $(6 a)-(6 g)$.

$$
\begin{aligned}
& S_{s}^{\min } \leq y_{s, t} \leq S_{s}^{\max }, \forall s \in \mathscr{S}, t \in \mathscr{T} \\
& y_{s, t}=y_{s, t-1}+r_{s, t}^{+} \eta_{s}^{+}-r_{s, t}^{-} / \eta_{s}^{-}, \forall s \in \mathscr{S}, t \in\{2, \ldots, T\} \\
& r_{s, t}^{+} \leq R_{s}^{+}, \forall s \in \mathscr{S}, t \in \mathscr{T} \\
& r_{s, t}^{-} \leq R_{s}^{-}, \forall s \in \mathscr{S}, t \in \mathscr{T} \\
& y_{s, T}=S_{s, 0}, \forall s \in \mathscr{S} \\
& y_{s, t}, r_{s, t}^{+}, r_{s, t}^{-} \geq 0, \quad \forall s \in \mathscr{S}, t \in \mathscr{T}
\end{aligned}
$$




$$
y_{s, 1}=S_{s, 0}+r_{s, 1}^{+} \eta_{s}^{+}-r_{s, 1}^{-} / \eta_{s}^{-}, \forall s \in \mathscr{S}
$$

The State Of Charge (SOC) of the ESS, the amount of energy stored in an ESS at certain period, is limited by the ESS technical lower and upper bounds, as modeled in (6a). The ESS can be charged or discharged in order to supply power to the grid or to absorb surplus of power if needed. This transaction is determined by the SOC of the ESS on the previous period, the charge and discharge efficiencies, and the rate of charge and discharge; this is modeled as a time balance constraint in (6b). The maximum amount of energy that can be charged or discharged from the ESS are modeled in (6c)-(6d). We consider that the final SOC of the battery at time $T$ must be the same as the initial SOC, as modeled in (6e). Non-negativity constraints for the SOC and the charge and discharge rate are modeled in (6f). Similarly to power generation case, for the initial time $t=1$, we assume that the ESS starts with a fixed SOC $S_{s, 0}$, that can be considered as a parameter; this is modeled in $(6 \mathrm{~g})$.

Power flow balance within the microgrid. Power generation, power flow, ESS charge and discharge, load demand, load shedding and photovoltaic generation are modeled as set of balance constraints, in (7a)-(7c).

$$
\begin{aligned}
& \sum_{e: j_{e}=i} f_{e}+\sum_{g: i_{g}=i} p_{g, t}+\sum_{s: i_{s}=i} r_{s, t}^{-}+\sum_{p v: i_{p v}=i} P V_{p v, t}=\left(D_{i, t}-q_{i, t}\right)+\sum_{e: i_{e}=i} f_{e}+\sum_{s: i_{s}=i} r_{s, t}^{+} \\
& q_{i, t} \leq D_{i, t}, \forall i \in \mathscr{I}, t \in \mathscr{T} \\
& q_{i, t} \geq 0, \forall i \in \mathscr{I}, t \in \mathscr{T}
\end{aligned}
$$

As modeled in (7a), the power flow that leaves from a bus, that is the sum of outgoing power flow, power generated, power discharged from the ESS and power generated by solar photovoltaic generators placed at bus $i$, must be equal to power flow that enters the same bus, given by the load demand (minus an amount that can be shed), the sum of incoming power flow, and the power charged to the ESS placed at bus $i$. Also the load that can be shed from a certain bus cannot be greater than the demand of this bus, as modeled in (7b). The non-negativity constraint for demand shedding is modeled in (7c).

Objective function for the operations of microgrid without renewable energy integration. For simplicity, we define the vectors $\mathbf{p}, \mathbf{x}, \mathbf{o}, \mathbf{f}, \mathbf{z}, \theta, \mathbf{p m}, \mathbf{m}, \mathbf{y}, \mathbf{r}, \mathbf{q}$, and $\mathbf{P V}$ to denote the compact form variables $p_{g, t}, x_{g, t}, c_{g, t}^{u / d}, f_{e, t}, z_{e, t}, \theta_{i_{e}, t}, p_{m, t}^{+/-/ F / N}$, $m_{t}, y_{s, t}, r_{s, t}^{+/-}, q_{i, t}$, and $P V_{p v, t}$, respectively.

For the operations of the microgrid, there are some elements that generate cost, including: power generation, startup and shut decisions for generators within the microgrid $\left(C_{g}^{p} p_{g, t}+c_{g, t}^{u}+c_{g, t}^{d}\right)$, cost of purchasing power from main grid under firm and non-firm contracts $\left(C_{m, t}^{-F} p_{m, t}^{-F}+C_{m, t}^{-N} p_{m, t}^{-N}\right)$, cost related to the lifetime reduction due to the use of ESS $\left(C_{s}^{+} r_{s, t}^{+}+C_{s}^{-} r_{s, t}^{-}\right)$, and cost related to load shedding $\left(C_{i, t}^{s h} q_{i, t}\right)$; on the other hand, revenues are obtained from the power sold to main grid $\left(C_{m, t}^{+F} p_{m, t}^{+F}+\right.$ $\left.C_{m, t}^{+N} p_{m, t}^{+N}\right)$. The objective function can be formulated in order to minimize the total cost, or to maximize the total profits. We formulate the objective function in order to maximize the total profits for the owner of the microgrid, as shown in (8). 


$$
\begin{aligned}
\max _{(\mathbf{p}, \mathbf{x}, \mathbf{o}, \mathbf{f}, \mathbf{z}, \theta, \mathbf{p m}, \mathbf{m}, \mathbf{y}, \mathbf{r}, \mathbf{q})} & \sum_{t \in \mathscr{T}}\left(C_{m, t}^{+F} p_{m, t}^{+F}+C_{m, t}^{+N} p_{m, t}^{+N}-\left(C_{m, t}^{-F} p_{m, t}^{-F}+C_{m, t}^{-N} p_{m, t}^{-N}\right)\right. \\
& -\sum_{g \in \mathscr{G}}\left(C_{g}^{p} p_{g, t}+c_{g, t}^{u}+c_{g, t}^{d}\right) \\
& \left.-\sum_{s \in \mathscr{S}}\left(C_{s}^{+} r_{s, t}^{+}+C_{s}^{-} r_{s, t}^{-}\right)-\sum_{i \in \mathscr{I}} C_{i, t}^{s h} q_{i, t}\right)
\end{aligned}
$$

Renewable energy uncertainty. We consider that the solar power generation, $P V_{p v, t}$, is random, in a way that the actual solar power output belongs to the uncertainty set $\mathscr{P} \mathscr{V}$. Assuming that the real data is within certain interval given by the mean and some boundaries (e.g., multiples of standard deviation) of the power generation for each time period and for each solar PV generator, we consider uncertainty set $\mathscr{P} \mathscr{V}$ as follows:

$$
\begin{array}{r}
P V_{p v, t} \in\left[\max \left\{P V_{p v, t}^{\text {mean }}-a_{p v, t} \sigma_{p v, t}, 0\right\}, \min \left\{P V_{p v, t}^{\text {mean }}+b_{p v, t} \sigma_{p v, t}, \overline{P V_{p v}}\right\}\right] \\
\forall p v, t \in \mathscr{T}
\end{array}
$$

where $P V_{p v, t}^{\text {mean }}, \sigma_{p v, t}$ and $\overline{P V_{p v}}$ denote the expected value, standard deviation, and technical upper bound for power generation, respectively, while $a_{p v, t}$ and $b_{p v, t}$ are nonnegative multiples for the standard deviation at the solar generator $p v$ at time $t$.

If we consider that the multiples of the standard deviation, $a_{p v, t}$ and $b_{p v, t}$ are bounded by 1 , the set in (9) can be explicitly formulated as shown in (10)

$$
\begin{aligned}
& P V_{p v, t}=P V_{p v, t}^{\text {mean }}-a_{p v, t} \sigma_{p v, t}+b_{p v, t} \sigma_{p v, t}, \quad \forall p v, t \in \mathscr{T} \\
& P V_{p v, t} \geq 0, \quad \forall p v, t \in \mathscr{T} \\
& P V_{p v, t} \leq \overline{P V_{p v}}, \quad \forall p v, t \in \mathscr{T} \\
& a_{p v, t}, b_{p v, t} \geq 0, \quad \forall p v, t \in \mathscr{T} \\
& a_{p v, t}, b_{p v, t} \leq 1, \quad \forall p v, t \in \mathscr{T}
\end{aligned}
$$

In order to keep the variation within an interval, a uncertainty budget (denoted as a constant $\Gamma$ ) is defined to bound the total number of multiples of standard deviation that the real power generation can be deviated from the expected value, i.e., it bounds the sum of the values of $a_{p v, t}$ and $b_{p v, t}$ as shown in (11):

$$
\sum_{p v} \sum_{t \in \mathscr{T}}\left(a_{p v, t}+b_{p v, t}\right) \leq \Gamma
$$

Note that $0 \leq \Gamma \leq|\mathscr{T}| \cdot\left(\sum p v\right)$, and that if we set $\Gamma=0$ there is no uncertainty on the solar PV generation and $P V_{p v, t}=P V_{p v, t}^{\text {mean }}$ for all PV generators and all periods of time.

Objective function for the operations of microgrid with renewable energy integration. In the following, we denote $\mathbf{p}$ for the compact form of the continuous variables $\mathbf{p}, \mathbf{o}, \mathbf{f}, \theta, \mathbf{p m}, \mathbf{y}, \mathbf{r}, \mathbf{q}$, and $\mathbf{b}$, for the compact form of the binary variables $\mathbf{x}, \mathbf{z}$ and $\mathbf{m}$. For robust optimization, we need to consider the worst-case scenario related 
to uncertain factors, i.e., solar PV power generation. This means, we need to look for the scenario in which the solar PV generation attains a minimum for total profits. The problem is formulated as a two-stage robust program. As explained in [24], in a two-stage robust optimization model the seconds-stage problem correspond to the decisions regarding the operations of the microgrid that are made in order to maximize profits, as presented in (8), after the uncertainty is revealed when the first-stage decisions are made. In other words, under the worst-case solar power generation, we want to maximize the operating profits of the owner of the microgrid. The two-stage robust optimization model is formulated in (12):

$$
\begin{array}{rl}
\min _{(\mathbf{P V} \in \mathscr{P} \mathscr{V})} \max _{\mathbf{b}, \mathbf{p}} & \mathbf{c}^{T} \mathbf{p} \\
\text { s.t. } & \mathbf{A b} \leq \mathbf{g} \\
\mathbf{D} \mathbf{p}-\mathbf{K} \mathbf{b} \leq \mathbf{h}-\mathbf{J} \cdot \mathbf{P V} & \mathbf{b} \in\{0,1\} \\
\mathbf{p} \geq \mathbf{0}
\end{array}
$$

where $\mathbf{c}, \mathbf{g}, \mathbf{h}, \mathbf{A}, \mathbf{D}, \mathbf{J}$, and $\mathbf{K}$ are the corresponding vectors or matrices representing the model coefficients for the defined constraints and objective function. The first set of constraints in (12) refer to constraints (1a)-(1f), (1m), (3c), and (5g). The second set of constraints correspond to (1g)-(11), (2a)-(2d), (3a), (4), (5a)-(5f), (6a)-(6g), and (7a)-(7c). The third and fourth set of constraints correspond to the binary and nonnegativity constraints for binary and continuous variables, respectively.

\section{Solution methodology and algorithm design}

The problem stated in (12) is difficult to solve, and we will need to do several reformulations for it in order to obtain a problem that can be solved. As demonstrated in [23], we can isolate the binary variables from the objective function to get a trilevel problem. This tri-level reformulation allow us to isolate the binary variables $\mathbf{b}$ from the continuous variables $\mathbf{p}$. Then, the original problem (12) can be formulated as shown in (13a)-(13e).

$$
\begin{aligned}
& \min _{(\mathbf{P V} \in \mathscr{P} \mathscr{V})} \max _{\mathbf{b}} \max _{\mathbf{p}} \mathbf{c}^{T} \mathbf{p} \\
& \text { s.t. } \mathbf{A b} \leq \mathbf{g} \\
& \mathbf{D} \mathbf{p}-\mathbf{K b} \leq \mathbf{h}-\mathbf{J} \cdot \mathbf{P V} \\
& \mathbf{b} \in\{0,1\} \\
& \mathbf{p} \geq \mathbf{0}
\end{aligned}
$$

Note that this reformulation also allow us to manage separately the set of constraints in (12), where (13b) correspond for those constraints only considering the set of integer variables $\mathbf{b}$, and (13c) consider the rest of the constraints that contains both integer and continuous variables, or only continuous variables. 
It can be seen that, for any set of generated PV power $\mathbf{P V}$, unit commitment $\hat{\mathbf{x}}$, line switching decisions $\hat{\mathbf{z}}$, and main grid connection decisions $\hat{\mathbf{m}}$ that are feasible to (12), the rest of the problem is a pure LP problem, which compact formulation is presented in (14). Complete formulation can be consulted on Appendix A.

$$
\begin{aligned}
& \max _{\mathbf{p}} \mathbf{c}^{T} \mathbf{p} \\
& \text { s.t. } \mathbf{D p} \leq \mathbf{h}+\mathbf{K} \hat{\mathbf{b}}-\mathbf{J} \cdot \mathbf{P} \hat{\mathbf{V}} \\
& \quad \mathbf{p} \geq \mathbf{0}
\end{aligned}
$$

Since (14) is a pure LP problem we can compute its corresponding dual problem, as presented in (15). Complete formulation for the dual can be consulted on Appendix B.

$$
\begin{gathered}
\min _{\alpha}(\mathbf{h}+\mathbf{K} \hat{\mathbf{b}}-\mathbf{J} \cdot \mathbf{P V})^{T} \alpha \\
\text { s.t. } \mathbf{D}^{T} \alpha \geq \mathbf{c} \\
\quad \alpha \geq \mathbf{0}
\end{gathered}
$$

Where $\alpha$ is the compact form representing the dual variables. If we replace the original third level pure LP problem with its dual, the original problem can be reformulated as (16), which is equivalent to (13a)-(13e) since strong duality of (14) holds.

$$
\begin{aligned}
\min _{(\mathbf{P V} \in \mathscr{P} \mathscr{V})} \max _{\mathbf{b}} \min _{\alpha}(\mathbf{h}+\mathbf{K b}-\mathbf{J} \cdot \mathbf{P V})^{T} \alpha \\
\text { s.t. } \mathbf{A b} \leq \mathbf{g} \\
\mathbf{D}^{T} \alpha \geq \mathbf{c} \\
\mathbf{b} \in\{0,1\} \\
\alpha \geq \mathbf{0}
\end{aligned}
$$

Note that we consider $\mathbf{b}$ now as decision variables. Also note that problem (16) is equivalent to (17).

$$
\begin{aligned}
& \min _{(\mathbf{P V} \in \mathscr{P} \mathscr{V}), \Theta} \Theta \\
& \text { s.t. } \Theta \geq \max _{\mathbf{b}} \min _{\alpha}(\mathbf{h}+\mathbf{K b}-\mathbf{J} \cdot \mathbf{P V})^{T} \alpha \\
& \mathbf{A} \mathbf{b} \leq \mathbf{g} \\
& \mathbf{D}^{T} \alpha \geq \mathbf{c} \\
& \mathbf{b} \in\{0,1\} \\
& \alpha \geq \mathbf{0}
\end{aligned}
$$

Where $\Theta$ is an auxiliary variable greater or equal than all the objective values for all possible feasible solutions with respect to $\mathbf{b}$ and $\alpha$. Theoretically we can solve 
this problem for any subsets of discrete variables $\hat{\mathbf{b}}^{(\omega)}, \omega=0, \ldots,|\mathscr{C}|$ that are feasible to (12), where $\mathscr{C}$ represents the set of all possible combinations for binary decision variables b. Then, problem (17) can be reformulated as (18).

$$
\begin{aligned}
& \min _{(\mathbf{P V} \in \mathscr{P} \mathscr{V}), \Theta} \Theta \\
& \text { s.t. } \Theta \geq \min _{\alpha^{(\omega)}}\left(\mathbf{h}+\mathbf{K} \hat{\mathbf{b}}^{(\omega)}-\mathbf{J} \cdot \mathbf{P V}\right)^{T} \alpha^{(\omega)} \\
& \mathbf{D}^{T} \alpha^{(\omega)} \geq \mathbf{c} \\
& \hat{\mathbf{b}}^{(\omega)} \in \mathscr{C} \\
& \alpha^{(\omega)} \geq \mathbf{0}
\end{aligned}
$$

Where $\hat{\mathbf{b}}^{(\omega)} \in \mathscr{C}$ are fixed values corresponding to all different feasible solution combinations for $\mathbf{b}$ in (12), and this allow us to remove the max operator and the set of constraints (13b) and (13d) from (17). Also note that, as $\alpha^{(\omega)}$ is the corresponding solution obtained for every $\omega \in \mathscr{C}$, we can merge the two min operators for the first and the third level. The tri-level model in (16) is reformulated as (19).

$$
\begin{aligned}
\min _{(\mathbf{P V} \in \mathscr{P} \mathscr{V}), \alpha^{(\omega)}, \Theta} & \Theta \\
\text { s.t. } \Theta \geq\left(\mathbf{h}+\mathbf{K} \hat{\mathbf{b}}^{(\omega)}-\mathbf{J} \cdot \mathbf{P V}\right)^{T} \alpha^{(\omega)} & \mathbf{D}^{T} \alpha^{(\omega)} \geq \mathbf{c} \\
& \hat{\mathbf{b}}^{(\omega)} \in \mathscr{C} \\
& \alpha^{(\omega)} \geq \mathbf{0}
\end{aligned}
$$

Note that $\mathbf{J} \cdot \mathbf{P V}^{T} \alpha$ is a bilinear term. In Appendix C, we prove this bilinear term corresponds to a product of a continuous and a binary variable, which is linearized using variables $\mathbf{U}^{(\omega)}$. After linearization, (19) is reformulated as (20).

$$
\begin{aligned}
\min _{\left(\mathbf{U}^{(\omega)}, \mathbf{P V} \in \mathscr{P} \mathscr{V}\right), \alpha^{(\omega)}, \Theta} & \\
\text { s.t. } & \Theta \geq\left(\mathbf{h}+\mathbf{K} \hat{\mathbf{b}}^{(\omega)}\right)^{T} \alpha^{(\omega)}-\mathbf{J}^{T} \mathbf{U}^{(\omega)} \\
& \mathbf{D}^{T} \alpha^{(\omega)} \geq \mathbf{c} \\
& \hat{\mathbf{b}}^{(\omega)} \in \mathscr{C} \\
& \alpha^{(\omega)} \geq \mathbf{0}
\end{aligned}
$$

C\&CG Formulation. Solving (20) for all the possible combinations of $\hat{\mathbf{b}}^{(\omega)} \in \mathscr{C}$ is not realistic. The C\&CG algorithm developed in [24] presents a methodology to solve this problem with only a subset of these combinations. We need to formulate the problem in a master problem - subproblem framework and solve it iteratively, 
where each iteration generates one combination of $\hat{\mathbf{b}}^{(\omega)} \in \mathscr{C}$, obtained by the subproblem, and is used by the master problem, adding a set of variables $\hat{\mathbf{b}}^{(\omega)}$ and the corresponding set of constraints to generate a solution for $\mathbf{P V}$. This algorithm has been proven to converge in finite number of iterations. For this purpose, we define the master problem as the one defined in (20), and the subproblem as the one defined in $(21)$

$$
\begin{aligned}
\underset{\mathbf{b}, \mathbf{p}}{\max } & \mathbf{c}^{T} \mathbf{p} \\
\text { s.t. } & \mathbf{A b} \leq \mathbf{g} \\
& \mathbf{D p}-\mathbf{K b} \leq \mathbf{h}-\mathbf{J} \cdot \mathbf{P} \hat{\mathbf{V}} \\
\mathbf{b} & \in\{0,1\} \\
\mathbf{p} & \geq \mathbf{0}
\end{aligned}
$$

Applying the $\mathrm{C} \& \mathrm{CG}$, the algorithm for solving this problem consists on the procedure shown in Table 4.

Table 4 C\&CG Algorithm

\begin{tabular}{|l|l|}
\hline 1: & Set $L B=-\infty, U B=\infty, \omega=0, \mathscr{C}=\emptyset$ \\
2: & While $U B-L B>\varepsilon$ \\
3: & Do Solve master problem $(\mathrm{MP})$ defined in $(20)$ \\
4: & Let $\mathbf{U}^{*}(\omega), \mathbf{P V}^{*}, \alpha^{*(\omega)}$ and $\Theta^{*}$ be MP optimal solution \\
5: & Update $L B=\Theta^{*}$ \\
6: & Solve subproblem $(\mathrm{SP})$ defined in (21) with $\mathbf{P V}=\mathbf{P V}^{*}$ \\
7: & Update $U B=\min \left\{U B, O b j^{*}(S P)\right\}$ \\
8: & Let $\mathbf{b}^{*}$ and $\mathbf{p}^{*}$ be $\mathrm{SP}$ optimal solution \\
9: & Let $\omega=\omega+1$ \\
10: & Update $\mathscr{C}=\mathscr{C} \cup\{\omega\}$ \\
11: & Set $\hat{\mathbf{b}}^{(\omega)}=\mathbf{b}^{*}$ \\
12: & End While \\
13: & Obtain optimal scheduling plan from SP \\
14: & End \\
\hline
\end{tabular}

\section{Numerical experiments}

The proposed formulations and algorithms were all implemented in $\mathrm{C}++$ and using CPLEX 12.3 via ILOG Concert Technology 2.9, and all computations were performed on a Linux machine with $4 \operatorname{Intel}(\mathrm{R}) \mathrm{Xeon}(\mathrm{TM}) \mathrm{CPU}$ 3.60GHz processors and 32GB RAM. Computational time is reported by CPU seconds.

For the IEEE 6-Bus Test System, the data taken from [28] (shown in Figure 1), was used. For this experiment, we assume a 7th node for the main grid. Thus, there are 7 transmission lines within the network, and a transmission line that connects the main grid and the microgrid at node 6 , as shown in Table 5. Additionally, there is one ESS in the network, placed in bus 6. ESS data was obtained from [16]. Information 
about ESS is shown in Table 6. The grid has three generators, and their information is shown in Table 7. The experiment is run over a 24 hours operation period.

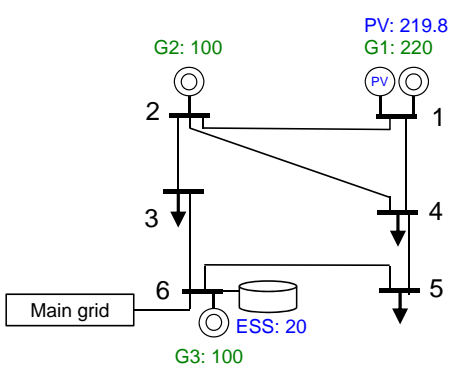

Fig. 1 IEEE 6-Bus Test System

Table 5 Information about transmission lines

\begin{tabular}{|c|c|c|c|c|}
\hline Line no. $(e)$ & $i_{e}$ & $j_{e}$ & $B_{e}$ & $F_{e}$ \\
\hline 1 & 1 & 2 & 5.88 & 200 \\
2 & 1 & 4 & 3.88 & 100 \\
3 & 2 & 4 & 5.08 & 100 \\
4 & 5 & 6 & 7.14 & 100 \\
5 & 3 & 6 & 55.56 & 100 \\
6 & 2 & 3 & 27.03 & 100 \\
7 & 4 & 5 & 27.03 & 100 \\
$8 e \in \mathscr{E}^{\prime}$ & 7 & 6 & 55.56 & 300 \\
\hline
\end{tabular}

Table 6 Information about ESS

\begin{tabular}{|c|c|c|c|c|c|c|c|c|c|}
\hline$i_{s}$ & $S_{s}^{\min }$ & $S_{s}^{\max }$ & $\eta^{+}$ & $\eta^{-}$ & $R_{s}^{+}$ & $R_{s}^{-}$ & $C_{s}^{+}$ & $C_{s}^{-}$ & $S_{s, 0}$ \\
\hline 6 & 4.4 & 20 & 0.9 & 0.9 & 16 & 16 & 3.5 & 3.5 & 10 \\
\hline
\end{tabular}

Table 7 Information about generators

\begin{tabular}{|c|c|c|c|c|c|c|c|c|c|c|c|c|}
\hline$g$ & $i_{g}$ & $C_{g}^{p}$ & $C_{g}^{u}$ & $C_{g}^{d}$ & $P_{g}^{\max }$ & $P_{g}^{\min }$ & $R_{g}^{d / u}$ & $\tilde{R}_{g}^{d / u}$ & $T_{g}^{u 0 / d 0}$ & $T_{g}^{u / d}$ & $\bar{p}_{g, 0}$ & $\bar{x}_{g, 0}$ \\
\hline 1 & 1 & 13.51 & 125 & 0 & 220 & 100 & 55 & 55 & 0 & 2 & 100 & 1 \\
2 & 2 & 32.63 & 249 & 0 & 100 & 10 & 50 & 50 & 0 & 2 & 10 & 1 \\
3 & 6 & 17.69 & 0 & 0 & 100 & 10 & 20 & 20 & 0 & 2 & 10 & 1 \\
\hline
\end{tabular}

Costs related to trade between main and microgrid were based on [29]. Cost of purchasing from main grid under firm contract, $C_{m, t}^{-F}$, are based on the off peak and on peak electricity cost. We assume that electricity traded above this amount is $30 \%$ more expensive, and also assume that microgrid can sell power to main grid at $80 \%$ 
of the price it can be purchased for power under firm contract, and 50\% for power above that quantity. Additionally, we assume that under firm contract, microgrid can purchase up to $100 \mathrm{~kW}$, in all the 24 periods, and sell up to $80 \mathrm{~kW}$ in periods $7-18$, and there is no firm contract for periods 1-6 and 19-24. Peak hours are from 16-20 hrs. During off-peak hours, costs are: $C_{m}^{-F}=60.30, C_{m}^{-N}=78.39, C_{m}^{+F}=48.24, C_{m}^{+N}=$ 30.15. During peak hours, costs are: $C_{m}^{-F}=100.60, C_{m}^{-N}=130.78, C_{m}^{+F}=80.48$, $C_{m}^{+N}=50.30$. Besides, we assume that for periods 1-6 and 19-24, the profits obtained from selling to the main grid are equal to zero, i.e., the main grid is not willing to pay for power during those periods. Also, we assume main grid has a big $M$ demand and supply, $300 \mathrm{~kW}$ for this case.

Demand information was taken from [28], but the hourly demand was approximated from demand behavior on [30], as shown in Table 8. Loads 1, 2 and 3 are placed on buses 3, 4 and 5, respectively. Costs for shedding demand are $C_{3, t}^{\text {sh }}=1000, C_{4, t}^{\text {sh }}=$ $1200, C_{5, t}^{s h}=800$, in all the 24 periods.

Table 8 Hourly demand

\begin{tabular}{|c|c|c|c|c|c|c|c|c|}
\hline Demand no. & 1 & 2 & 3 & 4 & 5 & 6 & 7 & 8 \\
\hline 1 & 35.34 & 34.17 & 32.99 & 31.81 & 30.63 & 30.63 & 34.17 & 37.70 \\
2 & 70.69 & 68.33 & 65.98 & 63.62 & 61.26 & 61.26 & 68.33 & 75.40 \\
3 & 29.55 & 28.56 & 27.58 & 26.59 & 25.61 & 25.61 & 28.56 & 31.52 \\
\hline Demand no. & 9 & 10 & 11 & 12 & 13 & 14 & 15 & 16 \\
\hline 1 & 44.77 & 51.84 & 58.91 & 60.09 & 68.33 & 69.51 & 69.51 & 69.51 \\
2 & 89.54 & 103.68 & 117.81 & 120.17 & 136.66 & 139.02 & 139.02 & 139.02 \\
3 & 37.42 & 43.33 & 49.24 & 50.23 & 57.12 & 58.11 & 58.11 & 58.11 \\
\hline Demand no. & 17 & 18 & 19 & 20 & 21 & 22 & 23 & 24 \\
\hline 1 & 69.51 & 67.15 & 60.09 & 58.91 & 58.91 & 57.73 & 54.19 & 42.41 \\
2 & 139.02 & 134.31 & 120.17 & 117.81 & 117.81 & 115.46 & 108.39 & 84.83 \\
3 & 58.11 & 56.14 & 50.23 & 49.24 & 49.24 & 48.26 & 45.30 & 35.45 \\
\hline
\end{tabular}

A PV generator with capacity $\overline{P V}=219.8 \mathrm{~kW}$ is placed in bus 1 . PV information was obtained from [31]. Hourly PV mean generation and standard deviation is shown in Table 9.

Table 9 Hourly RES generation

\begin{tabular}{|c|c|c|c|c|c|c|c|c|}
\hline Hour & 1 & 2 & 3 & 4 & 5 & 6 & 7 & 8 \\
\hline$P V^{\text {mean }}$ & 0 & 0 & 0 & 0 & 0 & 3.7366 & 60.445 & 130.341 \\
$\sigma$ & 0 & 0 & 0 & 0 & 0 & 1.030 & 6.260 & 9.708 \\
\hline Hour & 9 & 10 & 11 & 12 & 13 & 14 & 15 & 16 \\
\hline$P V^{\text {mean }}$ & 151.882 & 164.191 & 172.323 & 179.577 & 165.07 & 157.377 & 154.3 & 165.07 \\
$\sigma$ & 12.164 & 11.887 & 9.945 & 9.906 & 13.908 & 15.453 & 14.978 & 10.936 \\
\hline Hour & 17 & 18 & 19 & 20 & 21 & 22 & 23 & 24 \\
\hline$P V^{\text {mean }}$ & 147.925 & 98.690 & 43.740 & 0 & 0 & 0 & 0 & 0 \\
$\sigma$ & 10.302 & 9.232 & 4.477 & 0 & 0 & 0 & 0 & 0 \\
\hline
\end{tabular}

Finally, the budget of uncertainty was set to $\Gamma=10$, and the tolerance between upper and lower bound for $\mathrm{C} \& \mathrm{CG}$ algorithm was $\varepsilon=0.0001$.

After applying the above mentioned algorithm, the worst-case scenario for solar power generation is obtained, and the optimal decisions related to unit commitment, power generation, ESS dynamics, islanding operations and line switching under this 
scenario are made. The problem was solved in 4 iterations of the $\mathrm{C} \& \mathrm{CG}$ algorithm in 0.37 CPU seconds. Results are shown in Figures 2, 3, 4, and 5. Figure 2 shows the optimal power generation schedule. In this case, power generation of generator 1 varies during the 24 hours period, but it is always online. For generator 2, it is turned offline at period 1 and never turned online again. Generator 3 is online on periods 5-22, and offline the rest of the periods.
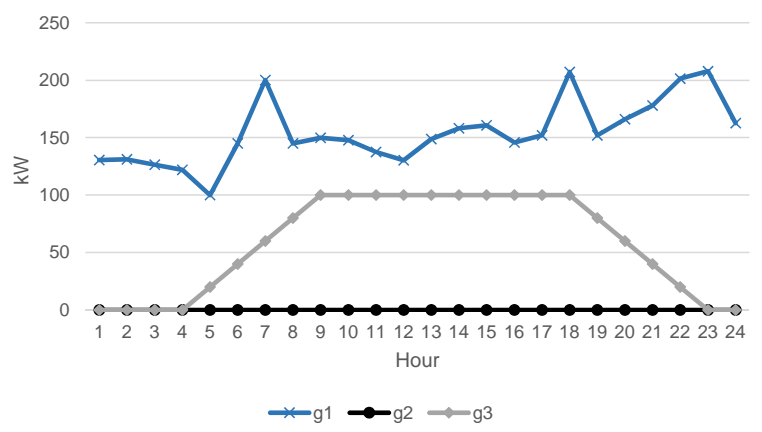

Fig. 2 Power generation

Figure 3 shows ESS optimal dynamics. The battery starts with a SOC of $4.4 \mathrm{kWh}$ at period 1 , then it reaches its maximum of $20 \mathrm{kWh}$ from periods $6-15$, and drops to its minimum from periods 16-18. It increases its SOC from periods 19-20 and reach the value of $10 \mathrm{kWh}$ from periods 21-24.

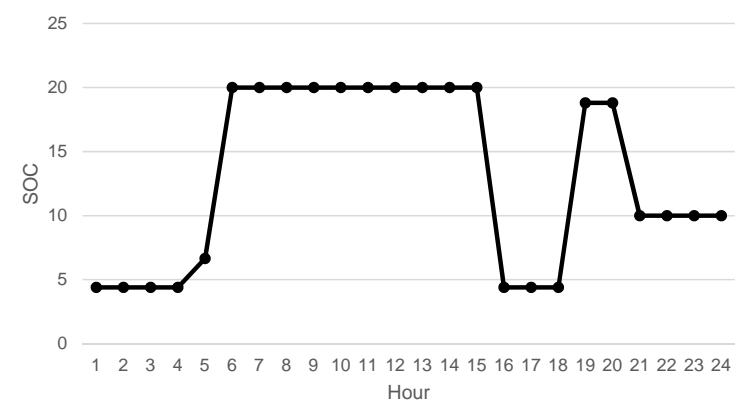

Fig. 3 State of Charge

Figure 4 shows optimal trade between main and microgrid. The microgrid does not buy or sell anything to main grid from periods 1-5 and 20-24, and works in islanding mode. The rest of the periods, the microgrid sells everything possible at the firm contract limit $(80 \mathrm{~kW})$, and sells different amount of power above that limit (in the non-firm contract). The microgrid does not buy power from the main grid.

In addition, figure 5 shows the aggregated total power supply, given by the sum of the power generation of DG and PV and the power discharged from the ESS at 


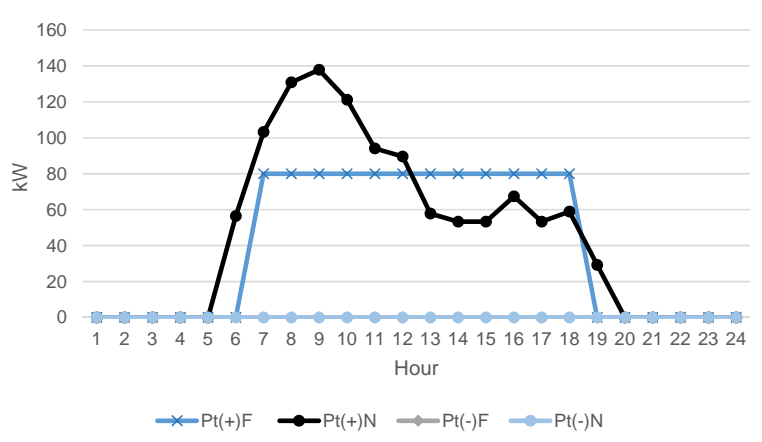

Fig. 4 Trade between main and microgrid

each period of time, and aggregated total power demand, given by the loads demand, the power sold to the main grid and the power charged to ESS. It can be seen that the total supply is greater than the loads demand, but the balance is obtained when power transferred to the main grid and power charged to ESS is considered. The total power generated by the DG was $5,106.52 \mathrm{~kW}$, while the total power supplied by the ESS was $27 \mathrm{~kW}$, and the total power generated by PV was $1,680.00 \mathrm{~kW}$. This is an integration rate of $24.66 \%$ of solar PV in this microgrid. Optimal objective value, in the worst-case scenario for solar PV generation, is $\$ 13,393$, which means that microgrid is obtaining profits based on it sales to the main grid.

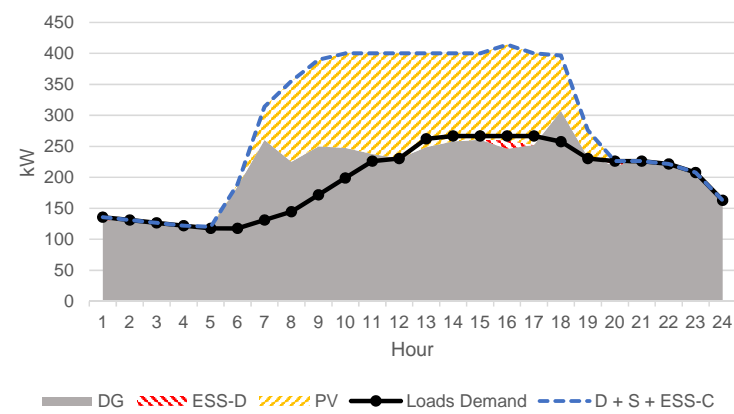

Fig. 5 Aggregated supply and demand

In order to quantify the performance of the algorithm, different instances were also tested: the IEEE 6-Bus, the IEEE RTS-96 [32], using one area as the microgrid, for a total of 24 buses, and the RTS-96 using two areas as a microgrid, for a total of 48 buses. Generators, demand, and lines information was taken from the RTS-96 system, with the exception of the costs related to power generating, start up/shutdown of generators, and load shedding, which have similar values than used on the 6-bus system before, as well as main grid-microgrid trade costs. In the case of RTS-96 with one area, five ESSs were located at buses 5, 9, 14, 19 and 24, each one with same capacities than the ESS used on the 6 buses system, and three solar PV generators 
were located at buses 1,9 and 17 , with capacity of 700,770 and $840 \mathrm{~kW}$, respectively. In the case of RTS-96 with two areas, nine ESSs were located at buses 6, 11, 16, 21, 26, 31, 36, 41 and 43, each one with same capacities than the ESS used on the 6 buses system. Also five solar PV generators were located at buses 1, 13, 15, 37 and 39 , with capacity of $700,770,700,770$ and $840 \mathrm{~kW}$, respectively. Different budgets of uncertainty were used for the three different systems, comparing the number of iterations, the CPU seconds required for each instance, and the integration of RES. Results are shown in Table 10.

Table 10 Comparison of the C\&CG algorithm for different instances

\begin{tabular}{|l|l|l|l|l|}
\hline Test System & $\Gamma$ & Iterations & CPU seconds & RES Integration (\%) \\
\hline & 2 & 2 & 0.22 & 25.95 \\
IEEE 6-Bus & 4 & 3 & 0.43 & 25.67 \\
& 6 & 3 & 0.33 & 25.28 \\
& 8 & 3 & 0.31 & 24.95 \\
& 10 & 4 & 0.37 & 24.66 \\
\hline & 5 & 1 & 9.47 & 29.72 \\
IEEE RTS-96-1 area & 10 & 1 & 1.73 & 29.52 \\
& 15 & 2 & 1.80 & 29.34 \\
& 20 & 2 & 2.23 & 29.18 \\
& 25 & 2 & 2.03 & 29.03 \\
\hline \multirow{5}{*}{ IEEE RTS-96-2 areas } & 5 & 2 & 5.06 & 25.63 \\
& 10 & 2 & 3.79 & 25.52 \\
& 15 & 1 & 3.83 & 25.42 \\
& 20 & 1 & 3.59 & 25.33 \\
& 25 & 2 & 3.68 & 25.22 \\
\hline
\end{tabular}

From the results it can be seen that the algorithm solves the problem in small number of iterations and CPU time for microgrids of 6, 24 and 48 buses, that include smart grid technologies. The optimal solution for the algorithm consists on the operations regarding DG, lines connectivity, ESS dynamics and islanding from the main grid. Results show that algorithm allows high RESs integration by searching for the worst-case scenario, and finding the optimal operations for the microgrid under this scenario.

\section{Conclusions}

In this paper, a model for optimal operations of a microgrid, including generator scheduling decisions, line connectivity decisions, power trade between main and microgrid, solar energy integration, and energy storage systems, is developed. A twostage robust optimization framework is used to manage the uncertainty of the solar power generation. Then, a reformulation was made for the min-max problem in order to apply the C\&CG algorithm. Finally, numerical experiments were performed on two power systems to illustrate how this model works, to find optimal decisions for operations of microgrid, under the worst-case scenario of solar energy generation. Future work can study the influences with the consideration demand response policies from the main grid. With advanced technologies for large-scale storage systems, 
their influences on renewable integration can also be studied.

Acknowledgements. J.L. Ruiz Duarte is supported by the Mexican National Council of Science and Technology (CONACYT) and the Mexican Department of Energy (SENER) for his PhD program. N. Fan is supported by University of Arizona Faculty Seed Grant (2016-2017).

\section{References}

1. T. Ackermann, G. Andersson, and L. Sder. Distributed generation: a definition. Electric Power Systems Research, 57(3):195 - 204, 2001.

2. M. Golari, N. Fan, and J. Wang. Two-stage stochastic optimal islanding operations under severe multiple contingencies in power grids. Electric Power Systems Research, 114:68 - 77, 2014.

3. M. Fürsch, S. Nagl and D. Lindenberger. Optimization of power plant investments under uncertain renewable energy deployment paths: a multistage stochastic programming approach. Energy Systems, 5(1):85 - 121, 2014

4. S.J. Pinto and G. Panda. Performance evaluation of WPT based islanding detection for grid-connected PV systems. International Journal of Electrical Power and Energy Systems, 78:537 - 546, 2016.

5. S. Okido and A. Takeda. Economic and environmental analysis of photovoltaic energy systems via robust optimization. Energy Systems, 4(3):239 - 266, 2013.

6. N. Fan, D. Izraelevitz, F. Pan, P.M. Pardalos, and J. Wang. A mixed integer programming approach for optimal power grid intentional islanding. Energy Systems, 3(1):77 - 93, 2012.

7. H.E. Brown, S. Suryanarayanan, S.A. Natarajan, and S. Rajopadhye. Improving reliability of islanded distribution systems with distributed renewable energy resources. IEEE Transactions on Smart Grid, 3(4):2028 - 2038, Dec 2012.

8. A. Maknouninejad et al. Cooperative control for self-organizing microgrids and game strategies for optimal dispatch of distributed renewable generations. Energy Systems, 3(1):23 - 60, 2012.

9. A.S.A. Awad, T.H.M. EL-Fouly, and M.M.A. Salama. Optimal ESS allocation and load shedding for improving distribution system reliability. IEEE Transactions on Smart Grid, 5(5):2339 - 2349, Sept 2014.

10. F. Genoese and M. Genoese. Assessing the value of storage in a future energy system with a high share of renewable electricity generation. Energy Systems, 5(1):19 - 44, 2014.

11. J. Siddiqui and E. Hittinger. Forecasting price parity for stand-alone hybrid solar microgrids: an international comparison. Energy Systems, DOI: 10.1007/s12667-017-0237-9, 2017.

12. A. Khodaei. Resiliency-oriented microgrid optimal scheduling. IEEE Transactions on Smart Grid, 5(4): 1584 - 1591, July 2014

13. J.A. Laghari, H. Mokhlis, M. Karimi, A.H.A. Bakar, and Hasmaini Mohamad. Computational intelligence based techniques for islanding detection of distributed generation in distribution network: A review. Energy Conversion and Management, 88:139 - 152, 2014.

14. T.M.L. Assis et al. Pilot field test of intentional islanding in distribution network. Energy Systems, 6(4):585-602, 2015.

15. R.B. Hytowitz and K.W. Hedman. Managing solar uncertainty in microgrid systems with stochastic unit commitment. Electric Power Systems Research, 119:111 - 118, 2015.

16. Y. Guo and C. Zhao. Islanding-aware robust energy management for microgrids. IEEE Transactions on Smart Grid, DOI: 10.1109/TSG.2016.2585092, 2016.

17. D. Zelazo, R. Dai, and M. Mesbahi. An energy management system for off-grid power systems. Energy Systems, 3(2):153 - 179, 2012.

18. W. Alharbi and K. Raahemifar. Probabilistic coordination of microgrid energy resources operation considering uncertainties. Electric Power Systems Research, 128:1 - 10, 2015.

19. A. Rezvani, M. Izadbakhsh and M. Gandomkar. Enhancement of microgrid dynamic responses under fault conditions using artificial neural network for fast changes of photovoltaic radiation and FLC for wind turbine. Energy Systems, 6(4):551 - 584, 2015.

20. B. Heymann et al. Continuous optimal control approaches to microgrid energy management. Energy Systems, DOI: 10.1007/s12667-016-0228-2, 2017. 
21. H. Wang and J. Huang. Joint investment and operation of microgrid. IEEE Transactions on Smart Grid, 8(2):833 - 845, March 2017.

22. A. Parisio, E. Rikos and L. Glielmo. A model predictive control approach to microgrid operation optimization. IEEE Transactions on Control Systems Technology, 22(5):1813 - 1827, 2014.

23. L. Zhao and B. Zeng. Vulnerability analysis of power grids with line switching. IEEE Transactions on Power Systems, 28(3):2727 - 2736, Aug 2013.

24. B. Zeng and L. Zhao. Solving two-stage robust optimization problems using a column-and-constraint generation method. Operations Research Letters, 41(5):457 - 461, 2013.

25. N. Kazemzadeh, S.M. Ryan and M. Hamzeei. Robust optimization vs. stochastic programming incorporating risk measures for unit commitment with uncertain variable renewable generation. Energy Systems, DOI: 10.1007/s12667-017-0265-5, 2017.

26. H. Vladimirou and S. A. Zenios. Stochastic Programming and Robust Optimization, pages $395-447$. Springer US, Boston, MA, 1997.

27. O. D. Melgar Dominguez et al. Optimal siting and sizing of renewable energy sources, storage devices, and reactive support devices to obtain a sustainable electrical distribution systems. Energy Systems, DOI: 10.1007/s12667-017-0254-8, 2017.

28. R.L. Chen, N. Fan, A. Pinar, and J.P. Watson. Contingency-constrained unit commitment with postcontingency corrective recourse. Annals of Operations Research, 249(1):381 - 407, 2017.

29. TEP. Tucson Electricity Power. Demand time-of-use, 2017. https : //www .tep.com/demand-tou/ (accessed April 26, 2017).

30. TEP. Tucson Electricity Power. 2017 integrated resource plan, 2017. https://www.tep.com/wp-content/uploads/2016/04/ TEP-2017-Integrated-Resource-FINAL-Low-Resolution.pdf (accessed April 26, 2017).

31. NREL. National Renewable Energy Laboratory. National solar radiation data base 1991- 2010 update, 2010. http://rredc.nrel.gov/solar/old_data/nsrdb/1991-2010/statistics/ hsf /722740_2010.hsf (accessed April 26, 2017).

32. C. Grigg et al. The IEEE reliability test system-1996. A report prepared by the reliability test system task force of the application of probability methods subcommittee. IEEE Transactions on Power Systems, 14(3):1010 - 1020, Aug 1999. 
Appendix A.

The third level for the optimization model is shown complete as follows

$$
\begin{aligned}
& \max _{(\mathbf{p}, \mathbf{f}, \mathbf{o}, \theta, \mathbf{p m}, \mathbf{y}, \mathbf{r}, \mathbf{q})} \sum_{t \in \mathscr{T}}\left(C_{m, t}^{+F} p_{m, t}^{+F}+C_{m, t}^{+N} p_{m, t}^{+N}-\left(C_{m, t}^{-F} p_{m, t}^{-F}+C_{m, t}^{-N} p_{m, t}^{-N}\right)\right. \\
& -\sum_{g \in \mathscr{G}}\left(C_{g}^{p} p_{g, t}+c_{g, t}^{u}+c_{g, t}^{d}\right) \\
& \left.-\sum_{s \in \mathscr{S}}\left(C_{s}^{+} r_{s, t}^{+}+C_{s}^{-} r_{s, t}^{-}\right)-\sum_{i \in \mathscr{I}} C_{i, t}^{s h} q_{i, t}\right) \\
& \text { s.t. }-c_{g, t}^{u} \leq-C_{g}^{u}\left(\hat{x}_{g, t}-\hat{x}_{g, t-1}\right), \forall g \in \mathscr{G}, t \in\{2, \ldots, T\} \\
& -c_{g, t}^{d} \leq-C_{g}^{d}\left(\hat{x}_{g, t-1}-\hat{x}_{g, t}\right), \forall g \in \mathscr{G}, t \in\{2, \ldots, T\} \\
& -c_{g, 1}^{u} \leq-C_{g}^{u}\left(\hat{x}_{g, 1}-\bar{x}_{g, 0}\right), \forall g \in \mathscr{G} \\
& -c_{g, 1}^{d} \leq-C_{g}^{d}\left(\bar{x}_{g, 0}-\hat{x}_{g, 1}\right), \forall g \in \mathscr{G} \\
& p_{g, t}-p_{g, t-1} \leq R_{g}^{u} \hat{x}_{g, t-1}+\tilde{R}_{g}^{u}\left(\hat{x}_{g, t}-\hat{x}_{g, t-1}\right)+P_{g}^{\max }\left(1-\hat{x}_{g, t}\right), \\
& \forall g \in \mathscr{G}, t \in\{2, \ldots, T\} \\
& p_{g, t-1}-p_{g, t} \leq R_{g}^{d} \hat{x}_{g, t}+\tilde{R}_{g}^{d}\left(\hat{x}_{g, t-1}-\hat{x}_{g, t}\right)+P_{g}^{\max }\left(1-\hat{x}_{g, t-1}\right), \\
& \forall g \in \mathscr{G}, t \in\{2, \ldots, T\} \\
& p_{g, 1} \leq \bar{p}_{g, 0}+R_{g}^{u} \bar{x}_{g, 0}+\tilde{R}_{g}^{u}\left(\hat{x}_{g, 1}-\bar{x}_{g, 0}\right)+P_{g}^{\max }\left(1-\hat{x}_{g, 1}\right), \quad \forall g \in \mathscr{G} \\
& -p_{g, 1} \leq-\bar{p}_{g, 0}+R_{g}^{d} \hat{x}_{g, 1}+\tilde{R}_{g}^{d}\left(\bar{x}_{g, 0}-\hat{x}_{g, 1}\right)+P_{g}^{\max }\left(1-\bar{x}_{g, 0}\right), \quad \forall g \in \mathscr{G} \\
& -p_{g, t} \leq-\hat{x}_{g, t} P_{g}^{\min }, \forall g \in \mathscr{G}, t \in \mathscr{T} \\
& p_{g, t} \leq \hat{x}_{g, t} P_{g}^{\max }, \forall g \in \mathscr{G}, t \in \mathscr{T} \\
& -f_{e, t} \leq \hat{z}_{e, t} F_{e}, \forall e \in \mathscr{E}, t \in \mathscr{T} \\
& f_{e, t} \leq \hat{z}_{e, t} F_{e}, \forall e \in \mathscr{E}, t \in \mathscr{T} \\
& -f_{e, t}+B_{e} \theta_{i_{e}}-B_{e} \theta_{j_{e}} \leq\left(1-\hat{z}_{e, t}\right) M_{e}, \forall e \notin \mathscr{E}^{\prime}, t \in \mathscr{T} \\
& f_{e, t}-B_{e} \theta_{i_{e}}+B_{e} \theta_{j_{e}} \leq\left(1-\hat{z}_{e, t}\right) M_{e}, \forall e \notin \mathscr{E}^{\prime}, t \in \mathscr{T} \\
& \left(p_{m, t}^{-N}+p_{m, t}^{-F}\right)-\left(p_{m, t}^{+N}+p_{m, t}^{+F}\right)-\sum_{e \in \mathscr{E}^{\prime}} f_{e, t} \leq 0, \forall t \in \mathscr{T} \\
& -\left(p_{m, t}^{-N}+p_{m, t}^{-F}\right)+\left(p_{m, t}^{+N}+p_{m, t}^{+F}\right)+\sum_{e \in \mathscr{E}^{\prime}} f_{e, t} \leq 0, \quad \forall t \in \mathscr{T} \\
& -p_{m, t}^{-N}-p_{m, t}^{-F} \leq \hat{m}_{t} \sum_{e \in \mathscr{E}^{\prime}} F_{e}, \forall t \in \mathscr{T} \\
& p_{m, t}^{-N}+p_{m, t}^{-F} \leq \hat{m}_{t} \sum_{e \in \mathscr{E}^{\prime}} F_{e}, \forall t \in \mathscr{T} \\
& -p_{m, t}^{+N}-p_{m, t}^{+F} \leq \hat{m}_{t} \sum_{e \in \mathscr{E}^{\prime}} F_{e}, \forall t \in \mathscr{T} \\
& p_{m, t}^{+N}+p_{m, t}^{+F} \leq \hat{m}_{t} \sum_{e \in \mathscr{E}^{\prime}} F_{e}, \forall t \in \mathscr{T} \\
& p_{m, t}^{-F} \leq \overline{P t}_{t}^{-F}, \forall t \in \mathscr{T}
\end{aligned}
$$




$$
\begin{aligned}
& p_{m, t}^{+F} \leq \overline{P t}_{t}^{+F}, \forall t \in \mathscr{T} \\
& -y_{s, t} \leq-S_{s}^{\min }, \forall s \in \mathscr{S}, t \in \mathscr{T} \\
& y_{s, t} \leq S_{s}^{\max }, \forall s \in \mathscr{S}, t \in \mathscr{T} \\
& y_{s, 1}-r_{s, 1}^{+} \eta_{s}^{+}+r_{s, 1}^{-} / \eta_{s}^{-} \leq S_{s, 0}, \quad \forall s \in \mathscr{S} \\
& -y_{s, 1}+r_{s, 1}^{+} \eta_{s}^{+}-r_{s, 1}^{-} / \eta_{s}^{-} \leq-S_{s, 0}, \quad \forall s \in \mathscr{S} \\
& y_{s, T} \leq S_{s, 0}, \forall s \in \mathscr{S} \\
& -y_{s, T} \leq-S_{s, 0}, \forall s \in \mathscr{S} \\
& y_{s, t}-y_{s, t-1}-r_{s, t}^{+} \eta_{s}^{+}+r_{s, t}^{-} / \eta_{s}^{-} \leq 0, \forall s \in \mathscr{S}, t \in\{2, \ldots, T\} \\
& -y_{s, t}+y_{s, t-1}+r_{s, t}^{+} \eta_{s}^{+}-r_{s, t}^{-} / \eta_{s}^{-} \leq 0, \forall s \in \mathscr{S}, t \in\{2, \ldots, T\} \\
& r_{s, t}^{+} \leq R_{s}^{+}, \forall s \in \mathscr{S}, t \in \mathscr{T} \\
& r_{s, t}^{-} \leq R_{s}^{-}, \forall s \in \mathscr{S}, t \in \mathscr{T} \\
& \sum_{e: j_{e}=i} f_{e}+\sum_{g: i_{g}=i} p_{g, t}+\sum_{s: i_{s}=i} r_{s, t}^{-}+q_{i, t}-\sum_{e: i_{e}=i} f_{e}-\sum_{s: i_{s}=i} r_{s, t}^{+} \\
& \leq D_{i, t}-\sum_{p v: i_{p v}=i} P \hat{V}_{p v, t}, \quad \forall i \in \mathscr{I}, t \in \mathscr{T} \\
& -\sum_{e: j_{e}=i} f_{e}-\sum_{g: i_{g}=i} p_{g, t}-\sum_{s: i_{s}=i} r_{s, t}^{-}-q_{i, t}+\sum_{e: i_{e}=i} f_{e}+\sum_{s: i_{s}=i} r_{s, t}^{+} \\
& \leq-D_{i, t}+\sum_{p v: i_{p v}=i} P \hat{V}_{p v, t}, \forall i \in \mathscr{I}, t \in \mathscr{T} \\
& q_{i, t} \leq D_{i, t}, \quad \forall i \in \mathscr{I}, t \in \mathscr{T} \\
& p_{g, t}, c_{g, t}^{u}, c_{g, t}^{d} \geq 0, \quad \forall g \in \mathscr{G}, t \in \mathscr{T} \\
& p_{m, t}^{-N}, p_{m, t}^{-F}, p_{m, t}^{+N}, p_{m, t}^{+F} \geq 0, \forall t \in \mathscr{T} \\
& y_{s, t}, r_{s, t}^{+}, r_{s, t}^{-} \geq 0, \forall s \in \mathscr{S}, t \in \mathscr{T} \\
& q_{i, t} \geq 0, \forall i \in \mathscr{I}, t \in \mathscr{T}
\end{aligned}
$$

\section{Appendix B.}

The dual problem for the third part of the tri-level problem can be written as

$$
\begin{aligned}
\min _{\alpha, \beta, \gamma, \delta, \zeta, l, \kappa, \lambda, \mu, v, \xi, \pi, \tau, \rho} & \sum_{g}\left(\alpha_{g, 1}\left(-C_{g}^{u}\left(\hat{x}_{g, 1}-\bar{x}_{g, 0}\right)\right)+\alpha_{g, 1}^{\prime}\left(-C_{g}^{d}\left(\bar{x}_{g, 0}-\hat{x}_{g, 1}\right)\right)\right) \\
& +\sum_{g} \sum_{t=2}^{T}\left(\alpha_{g, t}\left(-C_{g}^{u}\left(\hat{x}_{g, t}-\hat{x}_{g, t-1}\right)\right)\right. \\
& \left.+\alpha_{g, t}^{\prime}\left(-C_{g}^{d}\left(\hat{x}_{g, t-1}-\hat{x}_{g, t}\right)\right)\right) \\
& +\sum_{g}\left(\beta_{g, 1}\left(\bar{p}_{g, 0}+R_{g}^{u} \bar{x}_{g, 0}+\tilde{R}_{g}^{u}\left(\hat{x}_{g, 1}-\bar{x}_{g, 0}\right)+P_{g}^{\max }\left(1-\hat{x}_{g, 1}\right)\right)\right. \\
& \left.+\beta_{g, 1}^{\prime}\left(-\bar{p}_{g, 0}+R_{g}^{d} \hat{x}_{g, 1}+\tilde{R}_{g}^{d}\left(\bar{x}_{g, 0}-\hat{x}_{g, 1}\right)+P_{g}^{\max }\left(1-\bar{x}_{g, 0}\right)\right)\right)
\end{aligned}
$$


$+\sum_{g} \sum_{t=2}^{T}\left(\beta_{g, t}\left(R_{g}^{u} \hat{x}_{g, t-1}+\tilde{R}_{g}^{u}\left(\hat{x}_{g, t}-\hat{x}_{g, t-1}\right)+P_{g}^{\max }\left(1-\hat{x}_{g, t}\right)\right)\right.$

$\left.+\beta_{g, t}^{\prime}\left(R_{g}^{d} \hat{x}_{g, t}+\tilde{R}_{g}^{d}\left(\hat{x}_{g, t-1}-\hat{x}_{g, t}\right)+P_{g}^{\max }\left(1-\hat{x}_{g, t-1}\right)\right)\right)$

$+\sum_{g} \sum_{t}\left(-\gamma_{g, t} \hat{x}_{g, t} P_{g}^{\min }+\gamma_{g, t}^{\prime} \hat{x}_{g, t} P_{g}^{\max }\right)$

$+\sum_{e} \sum_{t}\left(\hat{z}_{e, t} F_{e}\left(\delta_{e, t}+\delta_{e, t}^{\prime}\right)\right)+\sum_{e \notin \mathscr{E}^{\prime}} \sum_{t}\left(1-\hat{z}_{e, t}\right) M_{e}\left(\zeta_{e, t}+\zeta_{e, t}^{\prime}\right)$

$+\sum_{t}\left(\boldsymbol{l}_{t}(0)+\boldsymbol{l}_{t}^{\prime}(0)\right)+\sum_{t}\left(\sum_{e \in \mathscr{E}^{\prime}} F_{e} \hat{m}_{t}\left(\kappa_{t}+\lambda_{t}+\kappa_{t}^{\prime}+\lambda_{t}^{\prime}\right)\right)$

$+\sum_{t}\left(\mu_{t} \overline{P t}_{t}^{-F}+\mu_{t}^{\prime} \overline{P t}_{t}^{+F}\right)+\sum_{s} \sum_{t}\left(-v_{s, t} S_{s}^{\min }+v_{s, t}^{\prime} S_{s}^{\max }\right)$

$+\sum_{s} S_{s, 0}\left(\xi_{s, 1}+\xi_{s, T+1}-\xi_{s, 1}^{\prime}-\xi_{s, T+1}^{\prime}\right)$

$+\sum_{s} \sum_{t=2}^{T}\left(\xi_{s, t}-\xi_{s, t}^{\prime}\right)(0)+\sum_{s} \sum_{t}\left(\pi_{s, t} R_{s}^{+}+\pi_{s, t}^{\prime} R_{s}^{-}\right)$

$+\sum_{i} \sum_{t}\left(\tau_{i, t} D_{i, t}\right)+\sum_{i} \sum_{t}\left(\rho_{i, t}-\rho_{i, t}^{\prime}\right)\left(D_{i, t}-\sum_{p v: i p v=i} P \hat{V}_{p v, t}\right)$

s.t. $-\alpha_{g, t} \geq-1, \forall g \in \mathscr{G}, t \in \mathscr{T}$

$-\alpha_{g, t}^{\prime} \geq-1, \quad \forall g \in \mathscr{G}, t \in \mathscr{T}$

$\beta_{g, t}-\beta_{g, t}^{\prime}-\beta_{g, t+1}+\beta_{g, t+1}^{\prime}-\gamma_{g, t}+\gamma_{g, t}^{\prime}+\rho_{i_{g}=i, t}-\rho_{i_{g}=i, t}^{\prime}$

$$
\geq-C_{g}^{p}, \quad \forall g \in \mathscr{G}, t \in\{1, \ldots, T-1\}
$$

$\beta_{g, T}-\beta_{g, T}^{\prime}-\gamma_{g, T}+\gamma_{g, T}^{\prime}+\rho_{i_{g}=i, T}-\rho_{i_{g}=i, T}^{\prime} \geq-C_{g}^{p}, \quad \forall g \in \mathscr{G}$

$-\delta_{e, t}+\delta_{e, t}^{\prime}-\zeta_{e, t}+\zeta_{e, t}^{\prime}+\rho_{j_{e}=i, t}-\rho_{j_{e}=i, t}^{\prime}$

$$
-\rho_{i_{e}=i, t}+\rho_{i_{e}=i, t}^{\prime}=0, \quad \forall e \notin \mathscr{E}^{\prime}, t \in \mathscr{T}
$$

$-\delta_{e, t}+\delta_{e, t}^{\prime}-l_{t}+\imath_{t}^{\prime}+\rho_{j_{e}=i, t}-\rho_{j_{e}=i, t}^{\prime}$

$-\rho_{i_{e}=i, t}+\rho_{i_{e}=i, t}^{\prime}=0, \forall e \in \mathscr{E}^{\prime}, t \in \mathscr{T}$

$B_{e} \zeta_{e, t}-B_{e} \zeta_{e, t}^{\prime}=0, \forall e \notin \mathscr{E}^{\prime}, t \in \mathscr{T}$

$-B_{e} \zeta_{e, t}+B_{e} \zeta_{e, t}^{\prime}=0, \quad \forall e \notin \mathscr{E}^{\prime}, t \in \mathscr{T}$

$\imath_{t}-\boldsymbol{l}_{t}^{\prime}-\kappa_{t}+\kappa_{t}^{\prime}+\mu_{t} \geq-C_{m, t}^{-F}, \quad \forall t \in \mathscr{T}$

$\imath_{t}-\imath_{t}^{\prime}-\kappa_{t}+\kappa_{t}^{\prime} \geq-C_{m, t}^{-N}, \quad \forall t \in \mathscr{T}$

$-l_{t}+l_{t}^{\prime}-\lambda_{t}+\lambda_{t}^{\prime}+\mu_{t}^{\prime} \geq C_{m, t}^{+F}, \quad \forall t \in \mathscr{T}$

$-\imath_{t}+\imath_{t}^{\prime}-\lambda_{t}+\lambda_{t}^{\prime} \geq C_{m, t}^{+N}, \quad \forall t \in \mathscr{T}$

$-v_{s, t}+v_{s, t}^{\prime}+\xi_{s, t}-\xi_{s, t}^{\prime}-\xi_{s, t+1}+\xi_{s, t+1}^{\prime} \geq 0$,

$\forall s \in \mathscr{S}, t \in\{1 \ldots, T-1\}$

$-v_{s, T}+v_{s, T}^{\prime}+\xi_{s, T}-\xi_{s, T}^{\prime}+\xi_{s, T+1}-\xi_{s, T+1}^{\prime} \geq 0, \forall s \in \mathscr{S}$

$-\eta_{s}^{+} \xi_{s, t}+\eta_{s}^{+} \xi_{s, t}^{\prime}+\pi_{s, t}-\rho_{i_{s}, t}+\rho_{i_{s}, t}^{\prime} \geq-C_{s}^{+}$,

$\forall s \in \mathscr{S}, t \in \mathscr{T}$ 


$$
\begin{aligned}
& \xi_{s, t} / \eta_{s}^{-}-\xi_{s, t}^{\prime} / \eta_{s}^{-}+\pi_{s, t}^{\prime}+\rho_{i_{s}, t}-\rho_{i_{s}, t}^{\prime} \geq-C_{s}^{-}, \\
& \forall s \in \mathscr{S}, t \in \mathscr{T} \\
& \tau_{i, t}+\rho_{i, t}-\rho_{i, t}^{\prime} \geq-C_{i, t}^{s h}, \quad \forall i \in \mathscr{I}, t \in \mathscr{T} \\
& \alpha_{g, t}, \alpha_{g, t}^{\prime}, \beta_{g, t}, \beta_{g, t}^{\prime}, \gamma_{g, t}, \gamma_{g, t}^{\prime} \geq 0, \forall g \in \mathscr{G}, t \in \mathscr{T} \\
& \delta_{e, t}, \delta_{e, t}^{\prime} \geq 0, \forall e \in \mathscr{E}, t \in \mathscr{T} \\
& \zeta_{e, t}, \zeta_{e, t}^{\prime} \geq 0, \forall e \notin \mathscr{S}^{\prime}, t \in \mathscr{T} \\
& \imath_{t}, \imath_{t}^{\prime}, \kappa_{t}, \kappa_{t}^{\prime}, \lambda_{t}, \lambda_{t}^{\prime}, \mu_{t}, \mu_{t}^{\prime} \geq 0, \quad \forall t \in \mathscr{T} \\
& v_{s, t}, v_{s, t}^{\prime}, \xi_{s, t}, \xi_{s, t}^{\prime}, \pi_{s, t}, \pi_{s, t}^{\prime} \geq 0, \forall s \in \mathscr{S}, t \in \mathscr{T} \\
& \tau_{i, t}, \rho_{i, t}, \rho_{i, t}^{\prime} \geq 0, \forall i \in \mathscr{I}, t \in \mathscr{T}
\end{aligned}
$$

\section{Appendix C.}

Linearization details. For reading purposes, some of the subindexes were removed in the following paragraphs. Note that, as $\left(\rho_{i}^{(\omega)}-\rho_{i}^{\prime(\omega)}\right)\left(D_{i}-\sum_{p v: i_{p v}=i} P V_{p v}\right)$ is a bilinear term, we need to use some linearization techniques in order to have a mixedinteger linear program. The uncertainty set $\mathscr{P} \mathscr{V}$, expressed in (10), only depending on variables $a_{p v, t} \in[0,1]$ and $b_{p v, t} \in[0,1]$, is a convex set. Then, the optimal value for $P V_{p v, t}$ must be an extreme points of this set, i.e. $a_{p v, t} \in\{0,1\}$ and $b_{p v, t} \in\{0,1\}$. Then, the extreme point of $P V_{p v, t}$ is given by

$$
\begin{aligned}
& P V_{p v}^{*}=P V_{p v}^{\text {mean }}-a_{p v} \sigma_{p v}+b_{p v} \sigma_{p v}, \forall p v \\
& a_{p v}, b_{p v} \in\{0,1\}, \forall p v
\end{aligned}
$$

Then, plugging (22) in the above mentioned bilinear term, we get

$$
\begin{aligned}
& \left(\rho_{i}^{(\omega)}-\rho_{i}^{\prime(\omega)}\right)\left(D_{i}-\sum_{p v: i_{p v}=i} P V_{p v}^{\text {mean }}\right)+ \\
& \sum_{p v: i_{p v}=i} \sigma_{p v}\left(\rho_{i}^{(\omega)} a_{p v}-\rho_{i}^{(\omega)} b_{p v}-\rho_{i}^{\prime(\omega)} a_{p v}+\rho_{i}^{\prime(\omega)} b_{p v}\right)
\end{aligned}
$$

The terms involving $\rho_{i}^{(\omega)} a_{p v}, \rho_{i}^{(\omega)} b_{p v}, \rho_{i}^{\prime(\omega)} a_{p v}$, and $\rho_{i}^{\prime(\omega)} b_{p v}$ are still nonlinear. They can be linearized by replacing $u_{p v}^{(\omega)}=\rho_{i: i_{p v}=i, t}^{(\omega)} a_{p v}, v_{p v}^{(\omega)}=\rho_{i: i_{p v}=i, t}^{(\omega)} b_{p v}, u_{p v}^{\prime(\omega)}=$ $\rho_{i: i_{p v}=i, t}^{\prime(\omega)} a_{p v}$, and $v_{p v}^{\prime(\omega)}=\rho_{i: i_{p v}=i, t}^{\prime(\omega)} b_{p v}$.

Then, (24) can be replaced by:

$$
\left(\rho_{i}^{(\omega)}-\rho_{i}^{\prime(\omega)}\right)\left(D_{i}-\sum_{p v: i_{p v}=i} P V_{p v}^{m e a n}\right)+\sum_{p v: i_{p v}=i} \sigma_{p v}\left(u_{p v}^{(\omega)}-v_{p v}^{(\omega)}-u_{p v}^{\prime(\omega)}+v_{p v}^{\prime(\omega)}\right)
$$

and adding the following constraints, that also must be included in the reformulated problem, as part of uncertainty set, such that $\mathbf{U}^{(\omega)}, \mathbf{P V} \in \mathscr{P} \mathscr{V}$ 
$u_{p v, t}^{(\omega)} \leq a_{p v, t} M, \quad \forall p v, t \in \mathscr{T}$

$u_{p v, t}^{(\omega)} \geq \rho_{i: i_{p v}=i, t}^{(\omega)}-M\left(1-a_{p v, t}\right), \quad \forall p v, t \in \mathscr{T}$

$u_{p v, t}^{(\omega)} \leq \rho_{i: i_{p v}=i, t}^{(\omega)}, \quad \forall p v, t \in \mathscr{T}$

$v_{p v, t}^{(\omega)} \leq b_{p v, t} M, \quad \forall p v, t \in \mathscr{T}$

$v_{p v, t}^{(\omega)} \geq \rho_{i: i_{p v}=i, t}^{(\omega)}-M\left(1-b_{p v, t}\right), \quad \forall p v, t \in \mathscr{T}$

$v_{p v, t}^{(\omega)} \leq \rho_{i: i p v}^{(\omega)}=i, t, \quad \forall p v, t \in \mathscr{T}$

$u_{p v, t}^{\prime(\omega)} \leq a_{p v, t} M, \forall p v, t \in \mathscr{T}$

$u_{p v, t}^{\prime(\omega)} \geq \rho_{i: i_{p v}=i, t}^{\prime(\omega)}-M\left(1-a_{p v, t}\right), \quad \forall p v, t \in \mathscr{T}$

$u_{p v, t}^{\prime(\omega)} \leq \rho_{i: i_{p v}=i, t}^{\prime(\omega)}, \quad \forall p v, t \in \mathscr{T}$

$v_{p v, t}^{\prime(\omega)} \leq b_{p v, t} M, \quad \forall p v, t \in \mathscr{T}$

$v_{p v, t}^{\prime(\omega)} \geq \rho_{i: i_{p v}=i, t}^{\prime(\omega)}-M\left(1-b_{p v, t}\right), \quad \forall p v, t \in \mathscr{T}$

$v_{p v, t}^{\prime(\omega)} \leq \rho_{i: i_{p v}=i, t}^{\prime(\omega)}, \quad \forall p v, t \in \mathscr{T}$

$b_{p v, t}, a_{p v, t} \in\{0,1\}, \forall p v, t \in \mathscr{T}$

$P V_{p v, t}=P V_{p v, t}^{\text {mean }}+\sigma_{p v, t}\left(-a_{p v, t}+b_{p v, t}\right), \quad \forall p v, t \in \mathscr{T}$

$P V_{p v, t} \geq 0, \forall p v, t \in \mathscr{T}$

$P V_{p v, t} \leq \overline{P V_{p v}}, \forall p v, t \in \mathscr{T}$

$\sum_{p v} \sum_{t \in \mathscr{T}}\left(a_{p v, t}+b_{p v, t}\right) \leq \Gamma$ 